\title{
Topotecan Decreases the Expression of Programmed Death-Ligand 1 in Glioblastoma Cell Lines; Implications for Immunotherapy
}

\author{
$\checkmark$ Correspondence \\ bernstockjd@ninds.nih.gov \\ 8 Disciplines \\ Oncology \\ Immunology \\ Q Keywords \\ Drug-Repurposing \\ Glioblastoma (GBM) \\ Programmed Death-Ligand 1 \\ (PD-L1) \\ Topotecan \\ Immunotherapy \\ t. Type of Observation \\ Standalone \\ o Type of Link \\ Standard Data
}

(O) Submitted Aug 25, 2017 (1) Published Oct 5, 2017

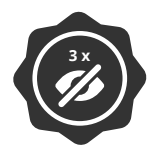

Triple Blind Peer Review The handling editor, the reviewers, and the authors are all blinded during the review process.

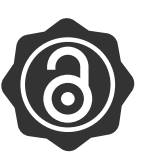

Full Open Access

Supported by the Velux Foundation, the University of Zurich, and the EPFL School of Life Sciences.

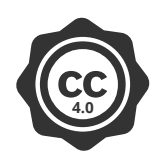

Creative Commons 4.0 This observation is distributed under the terms of the Creative Commons Attribution 4.0 International License.
Joshua D Bernstock, Daniel Ye, Florian A Gessler, Luca Peruzzotti-Jametti, Mark R Gilbert, Yves Pommier, Stefano Pluchino, Ichiro Nakano, John M Hallenbeck

Stroke Branch, National Institute of Neurological Disorders and Stroke (NIH), National Institutes of Health, Department of Clinical Neurosciences, Wellcome Trust-Medical Research Council Stem Cell Institute, University of Cambridge, Cambridge, UK; Stroke Branch, National Institute of Neurological Disorders and Stroke, National Institutes of Health; Department of Clinical Neurosciences, Wellcome Trust-Medical Research Council Stem Cell Institute, University of Cambridge, Department of Neurosurgery, Johann Wolfgang Goethe-Universität, Frankfurt am Main, Germany; Department of Clinical Neurosciences, Wellcome Trust-Medical Research Council Stem Cell Institute, University of Cambridge, Cambridge, UK; Neuro-Oncology Branch, National Cancer Institute and National Institute of Neurological Disorders and Stroke, National Institutes of Health; Developmental Therapeutics Branch and Laboratory of Molecular Pharmacology, Center for Cancer Research, NCI,; Department of Clinical Neurosciences, Wellcome Trust-Medical Research Council Stem Cell Institute, University of Cambridge, Cambridge, UK; Department of Neurosurgery, University of Alabama at Birmingham, Birmingham, AL, Comprehensive Cancer Center, University of Alabama at Birmingham, Birmingham, AL

\begin{abstract}
Glioblastoma (GBM) is the most aggressive primary brain tumor and thrives in a microenvironment of relative immunosuppression. The poor clinical outcome of these malignant tumors requires the development of novel treatment options/therapeutic regimens. Accordingly, numerous immunotherapies for GBM are currently being tested in ongoing clinical trials. Herein we have examined the ability of the FDA approved drug topotecan to suppress programmed death-ligand 1 (PD-L1) expression. Our results suggest a role for topotecan as an adjuvant therapy in treatment regimens targeting certain GBM patient subpopulations in whom the expression of PD-L1 has been confirmed.
\end{abstract}

\section{Introduction}

Glioblastoma (GBM) is the most common and aggressive primary tumor intrinsic to the central nervous system (CNS) being designated by the World Health Organization (WHO) as a grade IV glioma [1] [2]. The prognosis remains dismal despite a multimodal approach utilizing maximal surgical resection and adjuvant chemoradiation [3]. Therefore, novel therapies are urgently needed to improve outcomes for patients with this highly malignant neoplasm.

Evasion of the immune system is a hallmark of cancer, enabling tumor cells to escape immune surveillance [4]. Accordingly, therapeutic activation of the immune system through a variety of mechanisms is a rapidly expanding field in oncology, and numerous immune-activating agents have been approved by the FDA for cancer treatment over the last several years [5]. GBM tumors create an immunosuppressive microenvironment within the brain, resulting in both evasion of the body's immune system and support of the growth of cancer [6]

Cancer cells often express immune inhibitory signaling proteins that cause immune cell dysfunction and/or apoptosis. One of these inhibitory molecules is programmed death-ligand-1 (PD-L1), which binds to programmed death-1 (PD-1) expressed on Tcells, B-cells, dendritic cells, and natural killer T-cells to suppress anti-cancer immunity [7]. PD-L1 expression has been confirmed in GBM cells [8] [9] and has been linked to poor outcomes for GBM patients [10] [11]. It is prudent to note that PD-L1 expression has also been reported on microglia/macrophages in GBM specimens [12] [13].

Immune checkpoint inhibition targeting PD-L1 or PD-1 has been found to increase response rates in multiple cancer types [14] [15] [16] [17]. Considering the increasing evidence that immune checkpoint blockade and other immunotherapy strategies lead to sustained anti-tumor responses in several cancer types [18], there are increasingly more immunotherapy trials evaluating checkpoint inhibitors in patients with glioblastoma [19].

Hypoxia, which induces the stability of the transcription factor hypoxia-inducible factor-1-alpha (HIF-1 $\alpha)$ is a common feature of solid tumors [20] [21] [22]. Interestingly, recent reports have emerged demonstrating that HIF-1 $\alpha$ can drive the expression 
of PD-L1 [23] [24], leading to immunesuppression and tumor evasion [7]. HIF-1 $\alpha$ has also been shown to trigger angiogenesis in gliomas [25]. Moreover, activation of EGFR and downregulation of $\mathrm{P}_{53}$ and PTEN are seen in gliomas and have both been shown to affect HIF expression [26] [27] [28]. Of note, recent data show that chemotherapeutic agents used for GBM treatment, such as the topoisomerase I inhibitor topotecan, might have a suppressive effect on HIF-1 $\alpha$ [29] [30].

Until recently, it was unclear as to whether PD-L1 on tumor cells was sufficient for tumor immune evasion or simply correlated with an inflamed/hypoxic tumor microenvironment. The elegant work of Juneja et al. has now established reduced CD8 ${ }^{+} \mathrm{T}$-cell cytotoxicity as a key mechanism by which tumor PD-L1 suppresses antitumor immunity [31]. Accordingly, we sought to use the FDA approved small molecular topotecan to target the expression of PD-L1 in GBM in an effort to ultimately enhance the efficacy of targeted immunotherapies for this intractable CNS neoplasm.

\section{Objective}

To explore a role for topotecan as an adjuvant therapy in treatment regimens targeting certain GBM patient subpopulations in whom the expression of PD-L1 has been confirmed. 


\section{A}
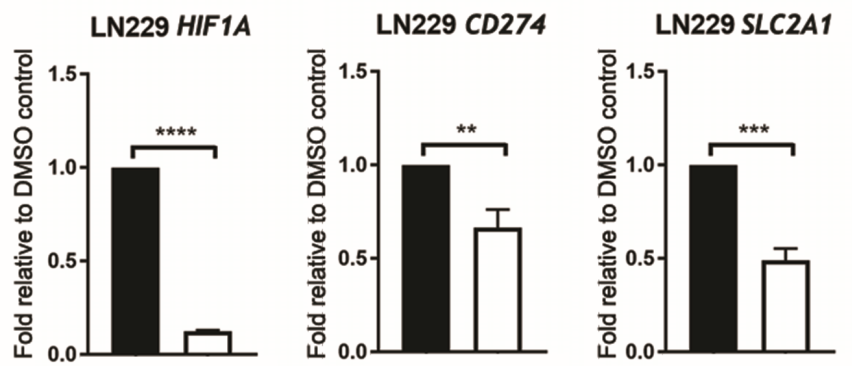

C
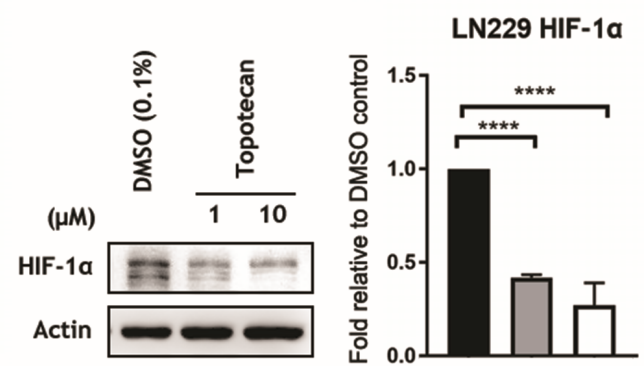

B

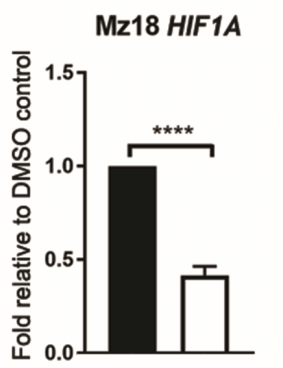

D
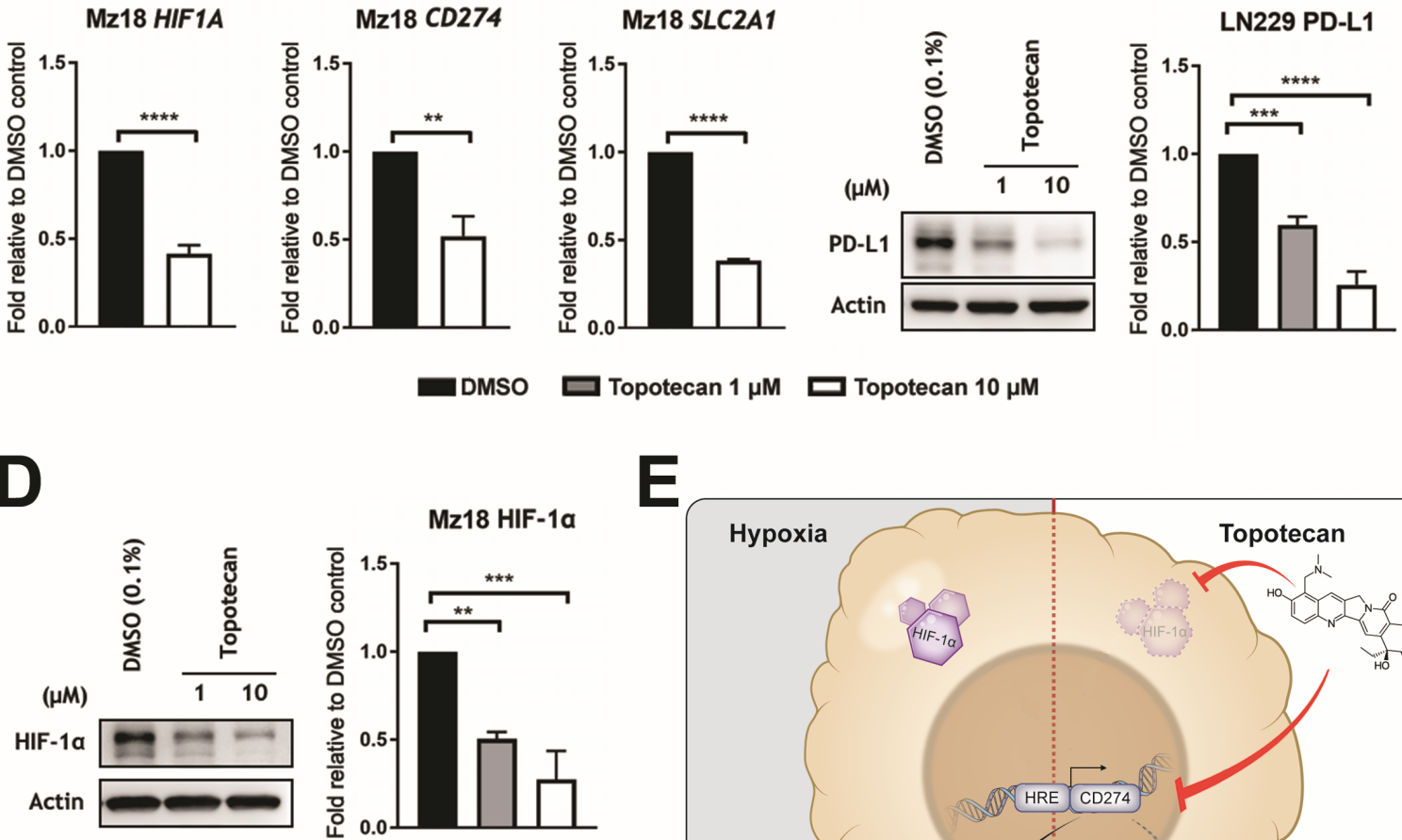

DMSO
Topotecan $10 \mu \mathrm{M}$

\section{E}

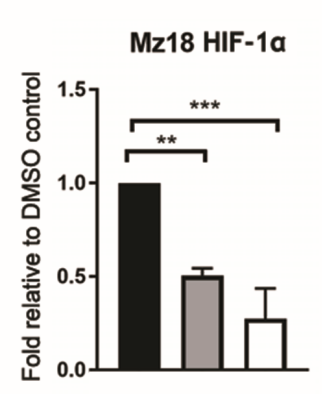

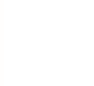

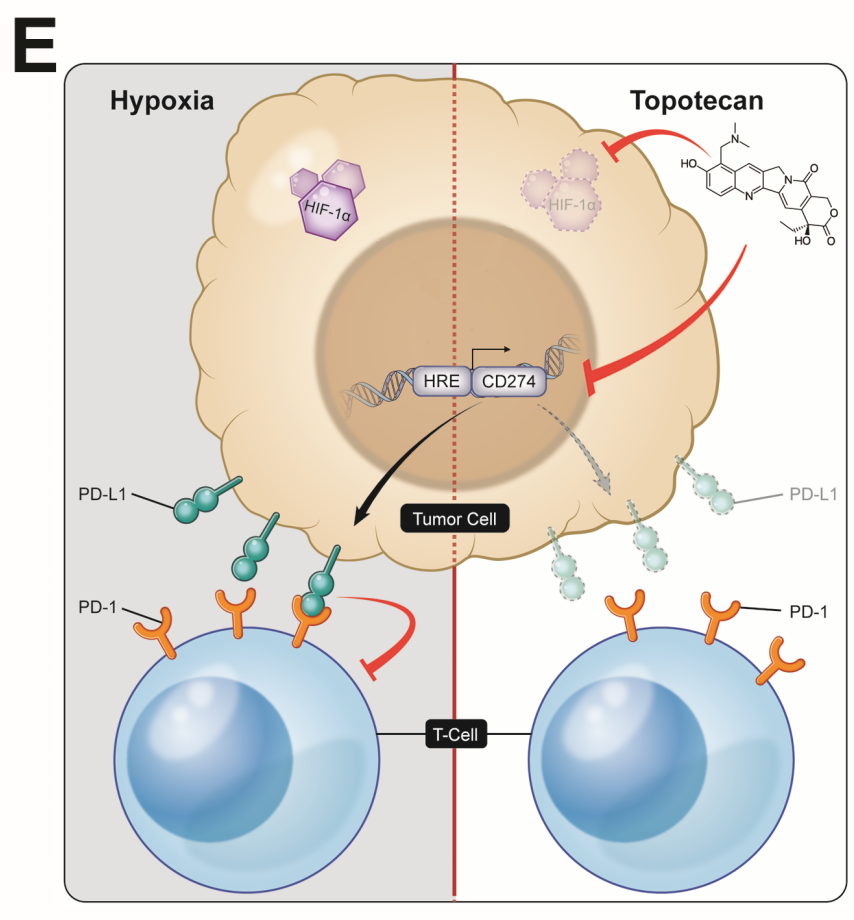

Figure Legend

Topotecan inhibits hypoxia-inducible factor 1-alpha (HIF-1 $\alpha)$ and programmed death-ligand-1 (PD-L1) in glioblastoma (GBM).

A. Treatment with $10 \mu \mathrm{M}$ topotecan significantly decreases levels of $H F_{1} A, C D_{274}$, and $S L C_{2} A 1$ mRNA transcripts in GBM cell line LN229. Expression fold change relative to the DMSO control was quantified using the $2^{-\triangle \triangle C T}$ method with $A C T B$ as the endogenous 
control. Means + /- SD from $N \geq 3$ independent experiments. ${ }^{* *} p<0.01,{ }^{* *} p<0.005$, $* * * * p<0.001$.

B. Treatment with $10 \mu \mathrm{M}$ topotecan significantly decreases levels of $H F_{1} A, C D_{2} 74$, and $S L C_{2} A 1$ mRNA transcripts in the GBM cell line Mz18. Expression fold change relative to the DMSO control was quantified using the $2^{-\Delta \Delta C T}$ method with ACTB as the endogenous control. Means $+/-$ SD from $N \geq 3$ independent experiments . ${ }^{* *} p<0.01$, $* * * * p<0.001$.

C. Treatment with topotecan significantly decreases levels of HIF- $1 \alpha$ and PD-L1 protein in GBM cell line LN229 at doses of $1 \mu \mathrm{M}$ and $10 \mu \mathrm{M}$. Representative immunoblots are shown. The bands corresponding to HIF-1 $\alpha(\sim 116-132 \mathrm{kDa})$ and PD-L1 $(\sim 40-50 \mathrm{kDa})$ were cropped in each lane and the total intensities were measured in ImageJ, normalized to the $\beta$-actin loading control, and expressed as a fold difference relative to the DMSO control. Means $+/-$ SD from $N \geq 3$ independent experiments. ${ }^{* * *} p<0.005,{ }^{* * * *} p<0.001$. D. Treatment with topotecan significantly decreases levels of HIF- $1 \alpha$ and PD-L1 protein in GBM patient cell line Mz18 at doses of $1 \mu \mathrm{M}$ and $10 \mu \mathrm{M}$. Representative immunoblots are shown. The bands corresponding to HIF- $1 \alpha(\sim 116-132 \mathrm{kDa})$ and PD-L1 $(\sim 40-50 \mathrm{kDa})$ were cropped in each lane and the total intensities were measured in ImageJ, normalized to the $\beta$-actin loading control, and expressed as a fold difference relative to the DMSO control. Means $+/$ - SD from $N \geq 3$ independent experiments . ${ }^{* *} p<0.01,{ }^{* * *} p<0.005$.

E. The binding of PD-L 1 to the cell surface receptor programmed cell death protein 1 (PD-1) suppresses CD8 ${ }^{+} \mathrm{T}$-cell cytotoxicity and permits immune evasion in tumor cells; topotecan inhibits PD-L1 expression.

\section{Results \& Discussion}

Herein we confirm that topotecan is an inhibitor of HIF- $1 \alpha$ in two human GBM cell lines, concordant with previous reports [29] [30]. Data obtained using topoisomerase I-resistant cell lines and inactive camptothecin analogs suggest that the targeting of topoisomerase I is critical to the inhibition of HIF-1 $\alpha$ [32]. Since this effect was abrogated via the interruption of RNA transcription [33], topotecan-induced perturbations of HIF- $1 \alpha$ may be occurring at the transcriptional level.

We therefore employed qPCR to determine whether the effects of topotecan occurred at the transcriptional level with regard to the reported decreases in HIF- $1 \alpha$, PD-L1, and GLUT1 (a canonical target of HIF-1 $\alpha$ ) [34]. We show that topotecan significantly decreased the expression of $H_{1} F_{1} A, C_{2} 74$, and $S L C_{2} A_{1}$ at a dose of $10 \mu \mathrm{M}$ in both the LN229 and Mz18 GBM cell lines (Figure A and B). To further investigate this effect, we conducted a dose-response study on HIF- $1 \alpha$ and PD-L 1 protein expression. We demonstrated that topotecan significantly decreases the expression of both HIF- $1 \alpha$ and PD-L 1 proteins at doses of $1 \mu \mathrm{M}$ and $10 \mu \mathrm{M}$ in both the LN229 and Mz18 GBM cell lines (Figure $\mathrm{C}$ and $\mathrm{D})$. Of note, such findings are in line with previous reports showing that HIF-1 $\alpha$ is capable of driving PD-L1 expression via binding to the hypoxia-response element (HRE) located within the promoter of $\mathrm{CD}_{274}$, the gene encoding PD-L1 [7] [24] (Figure E).

HIF- $1 \alpha$ activates the transcription of myriad genes that are involved in fundamental aspects of cancer biology (e.g. angiogenesis, cell survival, glucose metabolism, and/or invasion) [34]. Intratumoral hypoxia and genetic alterations can lead to HIF-1 overexpression, which has been associated with increased patient mortality in several cancer types [34]. This is becoming particularly apparent in GBM, a tumor characterized by pronounced areas of necrosis and hypoxia [20] [35]. Critically, our findings provide insight into a novel function of the camptothecin derivative topotecan (i.e. an inhibitor of HIF- $1 \alpha$ and PD-L1) in glioblastoma cells. Further studies investigating the effects of topotecan-mediated downregulation of PD-L1 (with or without a combination of checkpoint inhibitors) are needed to ultimately elucidate the clinical benefit of such a novel observation in GBM and other cancers.

\section{Conclusions}

Our findings suggest a role for topotecan as an adjuvant small molecule in immunotherapy regimens in GBM (and/or other cancers in which HIF- $1 \alpha$ and/or PD-L1 is expressed by the tumor) as an agent that may enhance the effect of immunomodulators such as 
checkpoint inhibitors. The authors therefore contend that the data presented suggest that repositioning topotecan may ultimately improve outcomes for GBM patients undergoing immunotherapy. Accordingly, we will seek to further this work in relevant animal models of GBM/immunotherapy.

\section{Limitations}

We understand and acknowledge that our work has limitations (i.e. we present solely in vitro data). Further, we rely on the literature with regard to the translational value which we assign our findings and remain uncertain if HIF- $1 \alpha$ is the sole driver of PD-L1 expression within our GBM cell lines. However, we are confident in the observations being put forward (i.e. topotecan decreased the levels of HIF-1 $\alpha$ and PD-L1) and feel they may ultimately be of translational value.

Our findings suggest a therapeutic role for the drug in GBM and other cancers, which express PD-L1 (i.e. with the understanding that topotecan has FDA approval for the treatment ovarian, cervical, and small cell lung cancers). It is therefore the authors' contention that the data presented suggest that adjuvant therapy with topotecan (i.e. drug repositioning) may ultimately improve outcomes for GBM patients undergoing immunotherapy. Accordingly, we will seek to further this work in relevant animal models of GBM/immunotherapy.

\section{Additional Information}

\section{Methods and Supplementary Material}

Please see https://sciencematters.io/articles/201709000008.

\section{Funding Statement}

This research was supported by the Intramural Research Program of the National Institute of Neurological Disorders and Stroke/National Institutes of Health (NINDS/NIH).

Ethics Statement

Not Applicable.

\section{Citations}

[1] Kleihues Paul et al. "Histopathology, classification, and grading of gliomas". In: Glia 15.3 (1995), pp. 211-221. DOI: 10.1002/glia.440150303. URL: https://doi.org/10.1002/glia.440150303.

[2] Louis David N. et al. "The 2016 World Health Organization Classification of Tumors of the Central Nervous System: a summary". In: Acta Neuropathologica 131.6 (2016), pp. 803-820. DOI: $10.1007 / \mathrm{s} 00401-016-1545-1$. URL: https://doi.org/10.1007/s00401-016-1545-1

[3] Stupp Roger et al. "Radiotherapy plus Concomitant and Adjuvant Temozolomide for Glioblastoma". In: New England fournal of Medicine 352.10 (2005), pp. 987-996. DOI: $10.1056 /$ nejmoa 043330 . URL https://doi.org/10.1056/nejmoa043330.

[4] Hanahan Douglas and Weinberg Robert A. "The Hallmarks of Cancer”. In: Cell 100.1 (2000), pp. 57-70. Dor: 10.1016/s0092-8674(00)81683-9. uRL: https : //doi.org/10.1016/s0092-8674(00)81683-9.

[5] Farkona Sofia, Diamandis Eleftherios P., and Blasutig Ivan M. "Cancer immunotherapy: the beginning of the end of cancer?" In: BMC Medicine 14.1 (2016). DOI: 10.1186/s12916-016-0623-5. URL: https://doi.org/10.1186/s12916-016-0623-5.
[6] Fecci Peter E. et al. "Increased Regulatory T-Cell Fraction Amidst a Diminished $\mathrm{CD}_{4}$ Compartment Explains Cellular Immune Defects in Patients with Malignant Glioma". In: Cancer Research 66.6 (2006), pp. 3294-3302. DoI:

10.1158/0008-5472. can-05-3773. uRL: https : //doi.org/10.1158/0008-5472.can-05-3773.

[7] Chen J. et al. "Regulation of PD-L1: a novel role of pro-survival signalling in cancer". In: Annals of Oncology 27.3 (2015), pp. 409-416. DoI: 10 . 1093/annonc/mdv615. URL: https://doi.org/10.1093/annonc/mdv615.

[8] Berghoff Anna Sophie et al. "Tumour-infiltrating lymphocytes and expression of programmed death ligand 1 (PD-L1) in melanoma brain metastases". In: Histopathology 66.2 (2014), pp. 289-299. DOI: 10 . 1111/his . 12537 . URL: https://doi.org/10.1111/his. 12537.

[9] Henrik Heiland Dieter et al. "Comprehensive analysis of PD-L1 expression in glioblastoma multiforme". In: Oncotarget (2017). DOI: 10.18632 /oncotarget . 15031. URL: https://doi.org/10.18632/oncotarget. 15031.

[10] Han Jiheun, Hong Yongkil, and Lee Youn Soo. "PD-L1 Expression and Combined Status of PD-L1/PD-1-Positive Tumor Infiltrating Mononuclear Cell Density Predict Prognosis in Glioblastoma Patients". In: fournal of Pathology and Translational Medicine 51.1 (2017), pp. 40-48. DOI: 10.4132/jptm. 2016.08.31. URL: https://doi.org/10.4132/jptm.2016.08.31. 
[11] Nduom Edjah K. et al. "PD-L1 expression and prognostic impact in glioblastoma". In: Neuro-Oncology 18.2 (2015), pp. 195-205. DOI: $10.1093 /$ neuonc/nov172. URL: https://doi.org/10.1093/neuonc/nov172.

[12] Preusser Matthias et al. "Clinical Neuropathology mini-review 6-2015: PD-L1: emerging biomarker in glioblastoma?” In: Clinical Neuropathology 34.11 (2015), pp. 313-321. DOI:

10.5414/np300922. URL:

https://doi.org/10.5414/np300922.

[13] Perng Powell and Lim Michael. "Immunosuppressive Mechanisms of Malignant Gliomas: Parallels at Non-CNS Sites". In: Frontiers in Oncology 5 (2015). DOI: 10.3389/fonc. 2015.00153. URL: https://doi.org/10.3389/fonc. 2015.00153.

[14] Callahan Margaret K., Postow Michael A., and Wolchok Jedd D. "CTLA-4 and PD-1 Pathway Blockade: Combinations in the Clinic". In: Frontiers in Oncology 4 (2015). DoI: $10.3389 /$ fonc .2014 .00385 . URL: https://doi.org/10.3389/fonc. 2014.00385.

[15] McDermott David et al. "Durable benefit and the potential for long-term survival with immunotherapy in advanced melanoma". In: Cancer Treatment Reviews 40.9 (2014), pp. 1056-1064. DOI: $10.1016 / \mathrm{j} . \mathrm{ctrv} .2014 .06 .012$. URL: https : //doi.org/10.1016/j.ctrv.2014.06.012.

[16] Mehta Kathan, Patel Keyur, and Parikh Rahul A. "Immunotherapy in genitourinary malignancies". In: fournal of Hematology and Oncology 10.1 (2017). DOI: 10.1186/s13045-017-0457-4. URL: https://doi.org/10.1186/s13045-017-0457-4.

[17] Somasundaram Ashwin and Burns Timothy F. "The next generation of immunotherapy: keeping lung cancer in check". In fournal of Hematology and Oncology 10.1 (2017). DOI: 10.1186/s13045-017-0456-5. URL: https://doi.org/10.1186/s13045-017-0456-5.

[18] Drake Charles G., Lipson Evan J., and Brahmer Julie R. "Breathing new life into immunotherapy: review of melanoma, lung and kidney cancer". In: Nature Reviews Clinical Oncology 11.1 (2013), pp. 24-37. DOI

10.1038/nrclinonc. 2013.208 . uRL: https: //doi.org/10.1038/nrclinonc.2013.208.

[19] Theeler Brett J. and Gilbert Mark R. "Advances in the treatment of newly diagnosed glioblastoma". In: BMC Medicine 13.1 (2015). DOI: $10.1186 / \mathrm{s} 12916-015-0536-8$. URL: https://doi.org/10.1186/s12916-015-0536-8.

[20] Colwell Nicole et al. "Hypoxia in the glioblastoma microenvironment: shaping the phenotype of cancer stem-like cells". In: Neuro-Oncology 19.7 (2017), pp. 887-896. DoI: 10.1093/neuonc/now258. URL: https://doi.org/10.1093/neuonc/now258.

[21] Semenza Gregg L. "HIF-1 mediates metabolic responses to intratumoral hypoxia and oncogenic mutations". In: fournal of Clinical Investigation 123.9 (2013), pp. 3664-3671. DOI: 10.1172/jCi67230. URL: https://doi.org/10.1172/jci67230.

[22] Semenza Gregg L. "Oxygen Sensing, Homeostasis, and Disease". In: New England fournal of Medicine 365.6 (2011), pp. 537-547. DoI: $10.1056 /$ nejmra1011165. URL: https://doi.org/10.1056/nejmra1011165.
[23] Barsoum I. B. et al. "A Mechanism of Hypoxia-Mediated Escape from Adaptive Immunity in Cancer Cells". In: Cancer Research 74.3 (2013), pp. 665-674. DOI: 10.1158/0008-5472 . can-13-0992. URL: https : //doi.org/10.1158/0008-5472.can-13-0992.

[24] Noman Muhammad Zaeem et al. "PD-L1 is a novel direct target of HIF-1 $\alpha$, and its blockade under hypoxia enhanced MDSC-mediated T cell activation". In: The fournal of Experimental Medicine 211.5 (2014), pp. 781-790. DOI: 10.1084/jem. 20131916. URL: https://doi.org/10.1084/jem. 20131916.

[25] Kaur B. "Hypoxia and the hypoxia-inducible-factor pathway in glioma growth and angiogenesis". In: Neuro-Oncology 7.2 (2005), pp. 134-153. DOI: $10.1215 / \mathrm{s} 1152851704001115$. URL https://doi.org/10.1215/s1152851704001115.

[26] Clarke K et al. "Mutant epidermal growth factor receptor enhances induction of vascular endothelial growth factor by hypoxia and insulin-like growth factor-1 via a $\mathrm{PI}_{3}$ kinase dependent pathway". In: British fournal of Cancer 84.10 (2001), pp. 1322-1329. DOI: 10.1054 /bjoc. 2001.1805 . URL: https://doi.org/10.1054/bjoc.2001.1805.

[27] Ravi et al. "Regulation of tumor angiogenesis by p53-induced degradation of hypoxia-inducible factor $1 \alpha$ ". In: Genes and Development 14.1 (2000), pp. 34-44. DOI: $10.1101 / \mathrm{gad} .14 .1 .34$.

[28] Zundel et al. "Loss of PTEN facilitates HIF-1-mediated gene expression". In: Genes and Development 14.4 (2000), pp. 391-396. DoI: $10.1101 / \mathrm{gad} .14 .4 .391$.

[29] Beppu Kiichiro et al. "Topotecan Blocks Hypoxia-Inducible Factor- $1 \alpha$ and Vascular Endothelial Growth Factor Expression Induced by Insulin-Like Growth Factor-I in Neuroblastoma Cells". In: Cancer Research 65.11 (2005), pp. 4775-4781. DoI: 10.1158/0008-5472. can-04-3332. URL: https : //doi.org/10.1158/0008-5472.can-04-3332.

[30] Rapisarda et al. "Identification of Small Molecule Inhibitors of Hypoxia-inducible Factor 1 Transcriptional Activation Pathway". In: Cancer Research 62.15 (2002), pp. 4316-4324.

[31] Juneja Vikram R. et al. "PD-L1 on tumor cells is sufficient for immune evasion in immunogenic tumors and inhibits CD8 T cell cytotoxicity". In: The fournal of Experimental Medicine 214.4 (2017), pp. 895-904. DOI: 10.1084/ j em. 20160801. URL: https://doi.org/10.1084/jem. 20160801.

[32] Rapisarda Annamaria et al. "Topoisomerase I-Mediated Inhibition of Hypoxia-Inducible Factor 1". In: Cancer Research 64.4 (2004), pp. $1475-1482$. DOI:

10.1158/0008-5472. can-03-3139. uRL: https : //doi.org/10.1158/0008-5472.can-03-3139.

[33] Rapisarda Annamaria, Shoemaker Robert H., and Melillo Giovanni. "Targeting Topoisomerase 1 to inhibit Hypoxia Inducible Factor 1". In: Cell Cycle 3.2 (2004), pp. 167-170. DOI: 10.4161/cc.3.2.688. URL: https://doi.org/10.4161/cc.3.2.688.

[34] Semenza Gregg L. "Targeting HIF-1 for cancer therapy". In: Nature Reviews Cancer 3.10 (2003), pp. 721-732. DOI: 10.1038/nrc1187. URL: https://doi.org/10.1038/nrc1187.

[35] Krex D. et al. "Long-term survival with glioblastoma multiforme". In: Brain 130.10 (2007), pp. 2596-2606. DOI: 10.1093/brain/awm204. URL: https://doi.org/10.1093/brain/awm204. 